\title{
Blockchain Applications in Health Care and Public Health: Increased Transparency
}

Pedro Elkind Velmovitsky ${ }^{1}$, BSc, MSc; Frederico Moreira Bublitz ${ }^{1,2}$, BSc, MSc, PhD; Laura Xavier Fadrique ${ }^{1}$, MSc, PMP; Plinio Pelegrini Morita ${ }^{1,3,4,5,6}$, PEng, MSc, PhD

\footnotetext{
${ }^{1}$ School of Public Health and Health Systems, University of Waterloo, Waterloo, ON, Canada

${ }^{2}$ Center for Strategic Technologies in Health (NUTES), State University of Paraiba (UEPB), Campina Grande, Brazil

${ }^{3}$ Institute of Health Policy, Management, and Evaluation, University of Toronto, Toronto, ON, Canada

${ }^{4}$ Research Institute for Aging, University of Waterloo, Waterloo, ON, Canada

${ }^{5}$ Department of Systems Design Engineering, University of Waterloo, Waterloo, ON, Canada

${ }^{6}$ eHealth Innovation, Techna Institute, University Health Network, Toronto, ON, Canada
}

\section{Corresponding Author:}

Plinio Pelegrini Morita, PEng, MSc, PhD

School of Public Health and Health Systems

University of Waterloo

200 University Ave W

Waterloo, ON, N2L 3G1

Canada

Phone: 115198884567 ext 41372

Email: plinio.morita@uwaterloo.ca

\section{Abstract}

Background: Although big data and smart technologies allow for the development of precision medicine and predictive models in health care, there are still several challenges that need to be addressed before the full potential of these data can be realized (eg, data sharing and interoperability issues, lack of massive genomic data sets, data ownership, and security and privacy of health data). Health companies are exploring the use of blockchain, a tamperproof and distributed digital ledger, to address some of these challenges.

Objective: In this viewpoint, we aim to obtain an overview of blockchain solutions that aim to solve challenges in health care from an industry perspective, focusing on solutions developed by health and technology companies.

Methods: We conducted a literature review following the protocol defined by Levac et al to analyze the findings in a systematic manner. In addition to traditional databases such as IEEE and PubMed, we included search and news outlets such as CoinDesk, CoinTelegraph, and Medium.

Results: Health care companies are using blockchain to improve challenges in five key areas. For electronic health records, blockchain can help to mitigate interoperability and data sharing in the industry by creating an overarching mechanism to link disparate personal records and can stimulate data sharing by connecting owners and buyers directly. For the drug (and food) supply chain, blockchain can provide an auditable log of a product's provenance and transportation (including information on the conditions in which the product was transported), increasing transparency and eliminating counterfeit products in the supply chain. For health insurance, blockchain can facilitate the claims management process and help users to calculate medical and pharmaceutical benefits. For genomics, by connecting data buyers and owners directly, blockchain can offer a secure and auditable way of sharing genomic data, increasing their availability. For consent management, as all participants in a blockchain network view an immutable version of the truth, blockchain can provide an immutable and timestamped log of consent, increasing transparency in the consent management process.

Conclusions: Blockchain technology can improve several challenges faced by the health care industry. However, companies must evaluate how the features of blockchain can affect their systems (eg, the append-only nature of blockchain limits the deletion of data stored in the network, and distributed systems, although more secure, are less efficient). Although these trade-offs need to be considered when viewing blockchain solutions, the technology has the potential to optimize processes, minimize inefficiencies, and increase trust in all contexts covered in this viewpoint. 
(JMIR Med Inform 2021;9(6):e20713) doi: 10.2196/20713

\section{KEYWORDS}

health care; blockchain; EHR; health insurance; drug supply chain; genomics; consent; digital ledger; food supply chain

\section{Introduction}

\section{Background}

Global society is moving into an age of ubiquitous and smart technologies that monitor our health, such as smart devices, Internet of Things solutions, and ambient assisted living systems. These technologies allow continuous and effortless health data collection at a previously unseen scale $[1,2]$, generating rich and massive data sets, known as big data [3].

The age of big data can lead to a change in the way health care is delivered. Generally, health care is reactive, in which individuals interact with health care services when there is something wrong [4,5] and usually to treat acute diseases, instead of proactive, in which real-time monitoring of health data from different sources leads to predictions and insights into individual and population health, as opposed to checkups with health services when a problem appears [4,5]. In this manner, a proactive and predictive health care model includes surveillance and monitoring of individuals through remote sensing technologies, such as smart bands and smart thermostats, generating large volumes of diverse and real-time data in a cost-effective manner. The use of such technologies in a community will also enable public health surveillance on a scale never seen before, allowing public health agencies to better understand the socioeconomic determinants of health and prevent disease outbreaks $[5,6]$.

However, to achieve this model of health care, there are challenges that need to be overcome. For example, health records are stored by different providers in systems that lack interoperability [7,8]. This makes data sharing difficult and prevents doctors from having a complete view of a patient's health [7,8]. Interoperability issues and costs also affect the availability of genomic data and minimize their benefits [9]. In addition, increasingly advanced methods of data collection and analysis of personal, medical, and genomic data raise concerns regarding ownership, privacy, and regulations of health data $[1,3]$.

One possible tool to overcome or mitigate these challenges is blockchain [6,10-13]. This technology can be seen as a distributed virtual ledger that records timestamped transactions $[6,12,13]$. Cryptography is used to ensure that when a block is added to the blockchain, it cannot be tampered with [12]. Hence, blockchain is a tamperproof digital ledger in which all participants view an immutable version of the truth, making it ideal to track an asset and enable trust among parties (eg, health data or user consent for data collection) $[6,7,12,14]$.

In 2016 and 2018, IBM Corporation surveyed more than 400 health care and life sciences executives on the use of blockchain technology. Among their findings, more than half of the executives in both industries had plans to adopt it by 2020 $[6,10,11]$. Given the perceived potential of blockchain by industry experts from multiple areas [6,10-12] and to help guide the implementation of digital solutions that can solve pressing needs in health care systems, the aim of this study is to review current blockchain solutions being developed by the health care industry. This paper provides a comprehensive view of the blockchain health care industry, providing guidance to innovators about how to leverage this technology in daily operations and how to implement solutions that can help evolve health care delivery. The COVID-19 outbreak has created an increased demand for home-based digital health solutions such as telehealth and telemonitoring [15], increasing the importance of using technologies such as blockchain to increase the transparency of digital transactions and data provenance [16,17].

\section{Related Work}

McGhin et al [18] provide an overview of the main opportunities and challenges for blockchain in the health care field and describe some initiatives (both in academia and industry) focused on developing blockchain solutions. Vazirani et al [19] detailed a systematic review examining the feasibility of blockchain for electronic health record (EHR) systems, finding several trade-offs that need to be considered during the design and development of blockchain. Trade-offs were further explored by O'Donoghue et al [20].

Farouk et al [13] provided a similar review to this one on the use of blockchain in the health care industry but mostly focused on its integration with Internet of Things devices and record management. Hasselgren et al [21] conducted a scoping review of blockchain in health care and, while focusing on peer-reviewed publications rather than the industry, they found that both the number and quality of blockchain research is growing.

Chukwu and Garg [22] provide a systematic review of blockchain applications specifically for the use of EHRs and health data sharing and do not focus on industry applications.

Agbo et al [23] conducted a systematic review of blockchain applications in health care, also focusing on academic literature, although some studies mention companies working with blockchain. The use cases found in this work are very similar to the use cases explored in this paper (suggesting a convergence between academia and industry research), but Agbo et al [23] found a predominance of studies focusing on EHRs when compared with other areas.

Most of these did not have an industry focus; rather, they usually discussed the computer science aspects of the technology or evaluated mostly academic work. In addition, as found by Agbo et al [23], most reviews focused on EHRs and not on additional use cases. Therefore, this review contributes to preview work by providing an overview of blockchain applications in the health care industry, while identifying what challenges and use cases are the current focus of health care companies working with blockchain. 


\section{Methods}

\section{Overview}

This narrative review [24] focuses on providing eHealth experts with a comprehensive narrative review of blockchain in health care. Blockchain is a novel technology that can provide increased transparency to data transactions in health care and public health $[6,10,11]$. Owing to its novelty and early stage implementation, significant development has been accomplished at the industry level, driving this review toward a combination of peer-reviewed academic literature and gray literature.

Our aim was to analyze blockchain in health care from an industry perspective, focusing on solutions developed by health and technology companies (although results from research and development initiatives and academia were used to complement knowledge when necessary).

Although not a scoping review, this paper followed the framework defined by Levac et al [25] for scoping reviews, ensuring that the findings were analyzed in a systematic manner. This framework consists of six stages: (1) identifying the research question (RQ); (2) identifying relevant studies; (3) selecting studies; (4) charting the data; (5) collating, summarizing, and reporting results; and (6) consultation (optional). In this narrative review, we leveraged phases 1-5.

\section{Identifying the RQ}

The primary objective is to identify how the health care industry views the potential of blockchain to solve current challenges. To fulfill this, two secondary goals need to be achieved: we must understand how blockchain works and the challenges facing the industry. Therefore, the following RQs were used to guide the reviews:

1. How do the blockchain systems work?

2. What are the current challenges faced by the health care industry today that can be addressed by blockchain technology?

3. For each of these challenges, which blockchain solutions are being developed by the health care industry?

\section{Identifying Relevant Studies}

Our review analyzes how the health care industry perceives the blockchain's potential to solve current challenges. To this end, we looked at gray literature in addition to traditional databases such as IEEE and PubMed, including search and news outlets such as Google Scholar, CoinDesk [26], CoinTelegraph [27], and Medium [28]. The keywords were a combination of "blockchain," "distributed ledger," "health," "industry," and "health care." Whenever possible, we looked at technical reports (usually available on companies' websites) in addition to news articles.

\section{Study Selection}

The primary exclusion criteria involved selecting solutions that address issues or challenges in health care. Blockchain solutions that only had applications in unrelated fields were not included. Additional restrictions included practical concerns regarding availability and language (only English references were included).

\section{Charting the Data}

To extract useful insights from the publications, we focused on two main types of information:

- What are the main health care challenges that the solution aims to improve?

- How is blockchain being used to improve the challenges?

More specifically, we looked at the main objective of the blockchain solution and the methods in which blockchain is being developed. Relevant bibliographical information, including title, authors, country, and year, was also extracted. This review focused on technical reports. If the technical report did not provide sufficient information, web articles were used to complement the results.

\section{Collating, Summarizing, and Reporting the Results}

Following the recommendations presented by Levac et al [25], the steps are as follows:

- Analysis: for each solution being presented, we mapped the challenges addressed and how blockchain is being used.

- Reporting results: after presenting additional information on blockchain, we will describe the challenge in question and its importance in health care, followed by a discussion on how blockchain is being used by the industry in this context.

- Implications for future research, practice, and policy: this final step will be addressed in the

Discussion

section, where we discuss the limitations of blockchain and additional concerns.

\section{Results}

\section{Overview}

We started this review by presenting relevant background information about blockchain, followed by an overview of the main challenges identified in our review: EHRs, supply chain, health insurance, genomics, and consent management. For each of these areas, we have also presented blockchain solutions developed by industry. Table 1 provides a summary of the results by describing each of the five identified challenges explaining how blockchain can offer a solution, along with examples. 
Table 1. Results of the literature review.

\begin{tabular}{|c|c|c|}
\hline Challenges & Description & Solutions \\
\hline $\begin{array}{l}\text { Electronic health } \\
\text { records }\end{array}$ & $\begin{array}{l}\text { Blockchain can provide an overarching framework } \\
\text { that allows transparent and auditable access to dis- } \\
\text { parate individuals' health records stored off-chain. } \\
\text { Patients would control data sharing parameters and } \\
\text { access. Some solutions also discuss integrating health } \\
\text { data from less traditional sources (eg, connected de- } \\
\text { vices) and the creation of a health data marketplace, } \\
\text { in which patients can sell their data to buyers through } \\
\text { crypto tokens }\end{array}$ & $\begin{array}{l}\text { - MedRec [7,8,29], PatientTruth [30,31], CareX [32,33], MEDIS } \\
\text { [34,35], GEM [36-40], MedicalChain [41,42], Humantiv and } \\
\text { Medoplex [43-45] }\end{array}$ \\
\hline Supply chain & $\begin{array}{l}\text { Blockchain can establish an immutable record of a } \\
\text { product's tracing throughout the supply chain. In the } \\
\text { case of health care, there have been many solutions } \\
\text { that implement a blockchain to track-and-trace drugs } \\
\text { and food products. In addition, smart contracts can be } \\
\text { used as monitoring and alert systems for proper trans- } \\
\text { port conditions (eg, a certain temperature range) }\end{array}$ & $\begin{array}{l}\text { Drug supply chain: BlockVerify [46-48], Merck [49,50], Modum } \\
\text { [51-54] } \\
\text { - Food supply chain: IBM Food Trust [55-58], Alibaba and Ant Fi- } \\
\text { nancial [59,60] }\end{array}$ \\
\hline Health insurance & $\begin{array}{l}\text { Smart contracts on the blockchain can potentially help } \\
\text { to settle health insurance claims and manage payment } \\
\text { in real time, making the process more efficient and } \\
\text { transparent for payers, providers, and patients. Other } \\
\text { potential use cases include pharmaceutical and medical } \\
\text { benefits, checks, and payment risk calculation }\end{array}$ & - PokitDok and DokChain [61-67], GEM [39], Payspan [68,69] \\
\hline Genomics & $\begin{array}{l}\text { Much like with electronic health records, blockchain } \\
\text { can provide a mechanism for controlling access to } \\
\text { separate existing data banks of genetic information. } \\
\text { In addition, blockchain can directly connect sellers of } \\
\text { genomic data-to-data buyers, creating a genomic data } \\
\text { marketplace. Data buyers could even provide rewards } \\
\text { for individuals to sequence their genomes, creating } \\
\text { their own data sets (eg, providing crypto tokens to in- } \\
\text { dividuals with a certain feature to be researched, in } \\
\text { return for their genomic information) }\end{array}$ & $\begin{array}{l}\text { - Nebula Genomics [9,70], LunaDNA [71-75], Shivom [76-79], } \\
\text { Zenome [80,81], EncrypGen [82-85], Macrogen [86-88] }\end{array}$ \\
\hline $\begin{array}{l}\text { Consent manage- } \\
\text { ment }\end{array}$ & $\begin{array}{l}\text { Blockchain can provide an immutable and timestamped } \\
\text { log of consent, allowing individuals to grant and re- } \\
\text { voke consent for different data types and periods. In } \\
\text { the case of health studies, it can also help researchers } \\
\text { to easily track, manage, and update user consent }\end{array}$ & $\begin{array}{l}\text { My31 app [89,90], Bitfury [91,92], HealthVerity Consent [93], } \\
\text { Verifiable Audit Trail (tracking of events related to health data) } \\
\text { [94-98], INSERM }{ }^{\mathrm{a}} \text { and APHP }{ }^{\mathrm{b}} \text { consent project [14], Queen's Uni- } \\
\text { versity BlockTrial [99], Patient Control and Consent Blockchain } \\
\text { initiative [100-102], Ubiquitous Health Technology Lab [6,103] }\end{array}$ \\
\hline
\end{tabular}

${ }^{a}$ INSERM: Institut National de la Santé Et de la Recherche Médicale.

${ }^{\mathrm{b}}$ APHP: Assistance Publique-Hôpitaux de Paris.

\section{What Is Blockchain?}

Blockchain is a virtual distributed ledger that records transactions among parties. It is operated by a network of computers in which each participant is called a node and possesses a copy of the ledger, regularly updated to ensure consistency. In other words, all nodes have access to the exact information $[12,18]$.

When a user makes a transaction, this transaction is timestamped and sealed in a block [12]. Through a consensus mechanism, this block is linked to previous existing blocks-hence the name blockchain. Different blockchains (eg, Bitcoin and Ethereum) have different consensus mechanisms [12]. A typical consensus mechanism, called Proof of Work, requires the nodes in the network to randomly guess a number that solves a mathematical puzzle; the first node to discover it seals the block. This process is called mining $[6,12,13,18]$.
The linkage between blocks is achieved through a method called hashing, in which new blocks point to the previous ones [12]. This technique converts data into a string of characters, called a hash. For example, a user may convert a text into the following hash: "f1abc234b79f6d6ay42a12c53468a1b13553r1r0fgr4039 rf08h958b5232b9n8." If a single character from this hash is changed, an entirely new string is generated. Although it is easy to generate a hash from a piece of information, it is impossible to discover the original information from a hash [12,22]. The Bitcoin blockchain hashes the nonce, alongside the transaction information and the hash of the preceding block. If a malicious party tries to tamper with information already stored in a block, the hash is altered, breaking the chain. This ensures that the blocks cannot be tampered with, and the information contained in the blockchain cannot be altered. Therefore, blockchain is a tamperproof digital ledger where all participants have access to an immutable version of the truth $[12,18]$. The flow of a transaction in the blockchain is shown in Figure 1. 
Figure 1. Flow of a transaction in the blockchain.

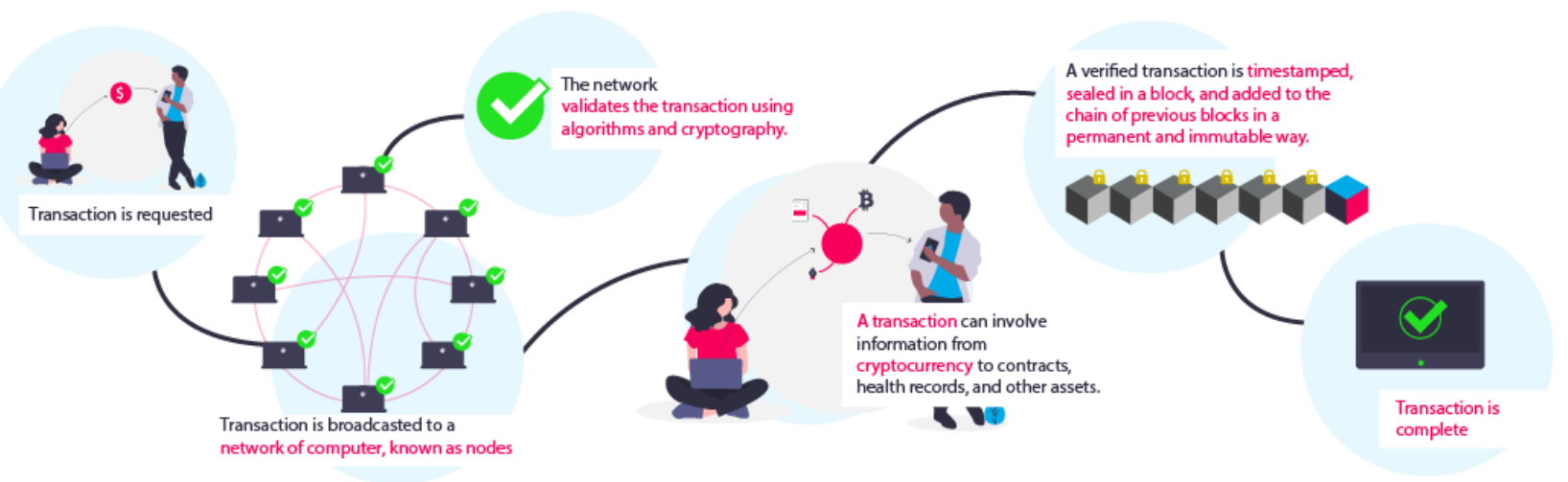

Blockchain is a type of distributed ledger technology, in which a consistent ledger is shared among parties to store a record, creating a distributed database. It is a distributed ledger technology that uses cryptographic and consensus mechanisms to increase trust [22].

There are also different types of blockchains. Although the nomenclature varies, they are usually defined as follows $[13,104,105]$ :

- Public blockchain: all participants can read and write new information to the ledger. Although new information can be added, no information can be deleted. Bitcoin is an example of a public blockchain.

- Permission (consortium and federated) blockchain: this is owned by a consortium of participants who define the permissions for joining and updating the network. For example, a consortium blockchain owned by health care providers can allow patients to change their information, but only providers may upload new information.

- Private blockchain: this is owned by a single entity that manages access, permission to read or write data, and even data deletion. Among the blockchain communities, some are of the opinion that private blockchains defeat the purpose of decentralized technologies by introducing a central authority.

From a health care perspective, one of the biggest concerns in capturing and coding patient information is privacy [106]. Several blockchain implementations allow the creation of smart, codified contracts that allow for the storage of immutable information. For example, Ethereum enabled the creation of smart contracts that codify contract agreements. When several parties agree to a transaction, they create mechanisms to ensure trust $[6,12,107]$. Smart contracts write the terms of a contract in code, which is executed on the blockchain, and has the ability to be self-executing and self-enforcing [12]. Therefore, smart contracts can minimize trust concerns among parties $[12,18,107]$.

Blockchain's features and design make it a model for processes plagued by trust issues [6,12,108], and it is ideal for increasing trust in contexts involving parties that do not have reason to trust each other $[6,12,108]$. One such context is health care [6].

\section{Blockchain in Health Care: Challenges and Solutions}

The following subsections describe a challenge in health care and discuss blockchain solutions being developed by companies to address them.

\section{Electronic Health Records}

\section{Challenge Description}

EHRs digitally store patients' health data $[6,7,109,110]$. However, data are fragmented throughout EHR systems: patients often interact with different health care providers (usually the stewards of the data), creating challenges related to accessing past information $[6,7,22]$. In addition, providers have different EHR systems that may not be fully interoperable [6,7]. These factors contribute to difficulties in data sharing [6,7].

Patients' health data end up in silos and cannot be integrated with data from other providers or sources, such as connected devices. Ultimately, there is no easy way to obtain a holistic view of a patient's health, leading to errors, delays, and poorer health outcomes $[6,7,18,110]$.

\section{Use of Blockchain}

Blockchain solutions can create an overarching hub, potentially on the cloud, to link all records of individual patients [7,8,18,29], without storing health data on the blockchain itself $[7,8,29,111]$. Rather, the blockchain infrastructure would act as a hub that points to the location of a patient's records off-chain [7,8,29]. Data access and changes to records can be tracked and displayed to the patient in real time. Furthermore, patients could control access to their records by giving permission to providers, researchers, and third parties to access their data. In this manner, an EHR-blockchain solution would allow for all health data from individuals to be accessed and controlled by the patient, facilitating a complete view of patients' health $[7,8,18,29]$. This solution would also give patients greater control and transparency over their health data [7,8,12,18,19,29].

For example, MedRec is a blockchain-enabled solution for EHRs [7,8,29]. It is a system developed by the Massachusetts Institute of Technology that provides a transparent view of medical history. MedRec uses smart contracts in Ethereum to encode metadata by referencing medical data from different sources, including information about ownership and permission. These references "create an accessible bread crumb trail for medical histor[ies]" [7]. Providers may append a new patient 
record in MedRec, but patients are the ones who give permission for data to be accessed and shared. This increases transparency and allows patients to keep track of their records $[7,8]$.

Similar solutions include PatientTruth [30,31], CareX [32,33], MEDIS [34,35], and MedicalChain [41,42]. Typically, EHR-blockchain solutions store references to off-chain files containing EHRs and also work with less traditional sources, such as data from connected devices. Patients control their records and with whom they wish to share their data (eg, health care professionals, hospitals, and insurance providers). Some of these solutions allow patients to sell deidentified records (eg, to health studies) with a crypto token from the platform. The financial component creates a form of health data marketplace in which patients own and are able to profit from their health data.

Another organization working with blockchain in an EHR context is CitizenHealth $[33,43]$, which developed two solutions: Humantiv [44], which also combines data from EHRs and other sources with an added gamification component in which patients earn rewards according to their health indicators, and Medoplex [45], the company's marketplace component. GEM, a US-based start-up, is developing a solution that uses Ethereum to create a shared network where providers have real-time access to medical documents [18]. GEM is partnering with Nordic-based Tieto to create a blockchain platform that enables patient control over medical records and genomic data [40,112,113].

It is important to note that a blockchain infrastructure, as described above, could mitigate data sharing issues by providing an interoperable, auditable, and secure landscape of transactions controlled by data owners. This, in turn, would allow easy and transparent access to disparate health records. As stated by McGhin et al [18], when discussing blockchain cloud infrastructures, "the role of blockchain in cloud data infrastructure is facilitating the creation of a decentralized and trusted cloud data provenance architecture that allows tamperproof records, greater transparency of data accountability, and enhanced privacy and availability of the data." However, the blockchain itself does not impact the interoperability of the health data itself or the local systems in which it is stored. Rather, it acts as an overarching infrastructure with references to off-chain resources whose access is auditable, secure, and transparent to all authorized parties within the distributed network.

\section{Drug Supply Chain}

\section{Challenge Description}

One of the biggest challenges faced by pharmaceutical companies today is counterfeit drugs. In total, US \$200 billion are lost to counterfeit drugs annually, and their use puts patients' lives at risk [46]. Manufacturers do not have a unified and interoperable system of supply chain management, lack incentives to share data and information, and are consequently siloed, making end-to-end traceability and drug provenance difficult $[46,114]$.

In the United States, the Drug Supply Chain Security Act (DSCSA) established a set of requirements that must be implemented by pharmaceutical companies until 2023. These requirements include product tracing and verification $[46,115,116]$.

\section{Use of Blockchain}

By storing transactional data from the supply chain on blockchain, it is possible to establish an immutable record of provenance [117]. Blockchain can provide a transparent ledger that traces products throughout the supply chain, from manufacturing to distribution. This will ensure compliance with the DSCSA and improve patient safety. Furthermore, blockchain can also track whether products are being transported and handled under appropriate conditions [111].

One of the companies working in this scenario was BlockVerify. The company is working on a DSCSA-compliant solution that traces products and identifies counterfeit drugs [46-48] A product is labeled with BlockVerify's tag and verified along the supply chain, with a permanent record on a private blockchain. Consumers and retail locations can use this record to ensure that the product is genuine. Merck has filed a patent to use blockchain to track drug information in the supply chain; while the company already has systems to prevent fraud, it is expected that blockchain could minimize existing inefficiencies $[49,50]$. Modum uses blockchain to ensure that medical products are being transported at the correct temperature [51-54]. Sensor devices are added to shipments, and smart contracts for each shipment are fixed with sensors, including the alarms. The sensors monitor the temperature during transportation and, when the shipment is received, the data are transferred to a blockchain and the smart contract evaluates whether conditions and regulations have been met [51-54].

Efforts have also been made to tackle the food supply chain. IBM Food Trust is a permissioned blockchain that allows stakeholders to view the supply chain history of a food item and complementary information (eg, certifications, testing, temperature, and location). For example, organizations can identify when a food item is contaminated and trace the contamination back to its source [55-58]. Another solution is being used in China: Ant Financial, an affiliate of the e-commerce enterprise Alibaba, is using a permissioned blockchain that tracks the production of rice in the city of Wuchang. Quick Response codes were added to rice packages, so that users scan these codes to obtain information (eg, location of the harvest, type of seed used, or transportation). Alibaba is also working on developing a food trust framework that, in its pilot phase, tracked shipments from China to Australia and New Zealand $[59,60]$.

\section{Health Insurance}

\section{Challenge Description}

There is a lack of trust between payers, providers, and patients with a complete view and coordination of health care [6]. Patients often pay expensive premiums while dealing with a lack of transparency and the ability to compare prices, in addition to the risk of insurance fraud that affects all stakeholders [32,41,69]. Providers must go through complex and bureaucratic processes to submit a claim [41]. The challenging ecosystem can be exemplified through the claims management process: (1) first, a provider must be covered by 
health insurers - meaning that the provider must also maintain benefits' databases and keep track of services delivered, adding additional expenses; (2) if patients receive services from the provider, the insurer checks the service against the patients' health plans to check their eligibility; (3) the process takes several weeks and involves multiple people checking agreements, leading to delays [41].

\section{Use of Blockchain}

In 2017, a survey revealed that $98 \%$ of payers with over 500,000 members are pursuing blockchain-enabled solutions [69]. Through the use of smart contracts, it is possible to codify terms of agreement between providers, payers, and patients, automating processes and minimizing inefficiencies. For example, PokitDok developed DokChain, a private blockchain that references off-chain file systems. DokChain contains several smart contracts that request and return data from health insurance providers and payers in real time [61-63], possibly enabling real-time status checks and mechanisms for error identification. The goal is for DokChain to make decisions on insurance claims in real time using smart contracts. As soon as a patient receives a service, it is recorded on DokChain and visualized by all stakeholders. Smart contracts can determine whose responsibility the claim falls under and how much is owed to each party, processing that amount in real time [62,64,65]. PokitDok also offers application programming interfaces to calculate payment risk for a patient [66] and to allow patients to schedule and pay for health services [67], automating payments and checking benefits in real time.

The start-up GEM created a blockchain prototype in 2017 that helped to settle a claim in less than 5 minutes [39]. Payspan, a 25-year-old health care reimbursement company, is also working on blockchain networks to connect providers and payers for claims management and payment processing [68,69].

Initiatives that focus on the creation of a health data marketplace (eg, Medoplex) want to allow health care buyers (eg, patients) and sellers (eg, providers) to connect without the need for insurance companies. Their goal is to create a marketplace platform where patients can search, select, and pay for health care services using a crypto token. CareX, MedicalChain, and BlockRx are other solutions that allow for the payment of health services with crypto tokens obtained from health record sharing $[32,41,46,111,114]$. Many companies that deal with EHRs seem to view health insurance as a complementary use case: by creating a health data marketplace platform that allows patients to share their data with health care providers and professionals, the idea seems to be that intermediaries (eg, insurance companies) will not be needed or their role will be diminished.

\section{Genomics}

\section{Challenge Description}

DNA is a molecule that encodes the genetic instructions of organisms [9], where genes are collections of DNA [118] and a genome is the collection of an organism's genes [9,119]. The human genome comprises more than 20,000 genes $[9,81,120,121]$.
The study of genomics data in conjunction with social and environmental determinants of health gave rise to precision medicine [122]. This field of research and treatment can help individuals and researchers better understand the cause of diseases, contribute to the development of new drugs, and aid in the creation of personalized interventions for individuals with specific genetic traits, in addition to many other benefits [123]. However, for this potential to be fully realized, large volumes of genomic data are required $[9,124]$.

There are several barriers to the availability of massive genomic data sets, including security and privacy concerns, prohibitive costs, and data sharing [9]. The latter is related to a lack of interoperability between systems that store genomic data and to data ownership [9]. In addition, a human genome generates over $200 \mathrm{~GB}$ of data, and it is estimated that more than 100 million genomes will be sequenced by 2025 . Therefore, storage and network transfer speeds also limit data sharing $[9,81,121]$.

\section{Use of Blockchain}

The business model for genomic data involves individuals hiring companies such as 23 andMe and Ancestry to sequence their genome and receive results. These companies then sell the sequenced data to researchers. With a blockchain network that connects data sellers (eg, an individual who sequenced their genome) and data buyers (eg, a pharmaceutical or research company), without the need for personal genomic companies acting as intermediaries, the transparency of the process will increase while costs will decrease. In addition, data sharing problems can be improved by connecting disparate genomic records on the blockchain, similar to what MedRec and other blockchain-EHR solutions have been proposing $[9,111]$. Although this will not solve all interoperability issues in the industry, it will allow easier, secure, and transparent access to disparate records of data, facilitating data sharing.

One of the companies that hope to build a genomic and health data marketplace is Nebula Genomics, through the development of a storage, sharing, and computing platform for biomedical big data [70]. The company has a partnership with Veritas Genomics. Veritas' platform processes and stores large amounts of genetic information, and Nebula hopes to build on top of it. Individuals will be able to store their genetic information on Veritas' platform and, with blockchain, share and sell their data in the genomic data marketplace. Similar to the case with EHRs, users own their data and control permissions for data sharing, increasing transparency and minimizing security and privacy concerns. Their proposed solution has the potential to [9] (1) minimize costs, since data buyers will acquire data directly from owners who can receive sequencing subsidies from buyers to encourage the generation of genomic data sets (eg, offering sequencing subsidies to individuals with specific traits that a specific research group plans to study); (2) increase transparency, since owners will control access and data sharing will be protected through cryptography. Owners will remain anonymous, while buyers will have to provide information about their identity. Blockchain records all transactions in an immutable log that is easily auditable; and (3) increase the availability of data by integrating data from several sources. The network offers space-efficient data-encoding formats and 
leverages computer resources to facilitate the transfer of information [9,70,121].

Other companies have similar solutions, with minor differences. LunaDNA allows anyone to join the blockchain network and win company shares based on shared data in the form of a crypto token. Researchers pay to conduct research on aggregated data, and proceeds earned from research are passed on to stakeholders as dividends. Unlike Nebula Genomics, LunaDNA currently does not provide genome sequencing services, but it accepts files from companies such as 23andMe and Ancestry. Blockchain is used to give individuals ownership of their data and for the generation of an immutable log of transactions [71-75]. Shivom [76-79], Zenome [80,81], EncrypGen [82-85], and Macrogen [86-88] are other similar companies that are using blockchain to empower patients by allowing them to own and share genomic data.

\section{Consent Management}

\section{Challenge Description}

In health studies, the process of obtaining consent from participants is fallible [125]. The Food and Drug Administration cited the main deficiencies related to consent: the failure to obtain informed consent, use of expired or incomplete forms, failure to provide copies of the forms to participants, missing documents, and changes made to documents without the approval of a review ethics board [14,125]. These problems are aggravated given that reconsent has to be sought in several cases (eg, when there is a revision of the study protocol, new risks are discovered, or there is a worsening of the medical condition of a participant) [126]. Oftentimes, consent needs to be obtained for cohabitants, caregivers, or legal guardians [125-128].

In addition to limitations in traditional consent methods, the global society is moving into an age of ubiquitous smart technologies that monitor our health, which increases the complexity of data collection points and, in turn, of consent management. The challenge of obtaining consent for increasingly advanced methods of data collection, use, and disclosure calls for new solutions to perfect consent procedures and the need to protect the safety of individuals.

\section{Use of Blockchain}

Blockchain can provide an immutable and timestamped log of consent, making the process more transparent $[6,12,14,111,129]$. In the case of health studies, participants will be able to monitor and manage consent, giving informed consent for certain types of data to be collected but not others and revoking their consent at any time. Through the use of cryptographic techniques of identity management, participants can make sure that they are reviewing the latest consent forms and that these were approved by the review ethics board. Researchers would also be benefited, as the measures taken to ensure ethical and legal requirements throughout the research would be clearly auditable. Moreover, it would be easier for them to obtain, track, and update patient consent during the study.

With a consent management platform built on blockchain, authorized parties in the network will have access to timestamped and tamperproof logs of user consent. However, this does not serve as a magic bullet to solve all consent management issues, and researchers must still be careful when collecting informed consent and ensure all ethical and legal requirements. For example, participants may revoke their consent at any time during the study (revocability), and researchers would need to stop collecting participant data at this point despite the fact that data may already have been collected (nonretractability). Similar to traditional health studies, consent forms should include a description of the protocols necessary for revoking consent according to data protection regulations and indicate to participants whether their data are being deleted after their consent is nullified. For example, Article 17 of the General Data Protection Regulation describes the "right to be forgotten" [130], in which personal data must be deleted after consent is revoked.

This would not present a problem for blockchain systems, as the collected data would not be stored on the blockchain, rather the consent of the individual is. If a participant revokes consent, the system will instantly be updated to reflect this and notify the researchers of the change. All consent status updates of participants cannot be tampered with on the blockchain and can be easily auditable in case of problems [129]. Furthermore, if the study uses connected devices for data collection, smart contracts can be developed to ensure that, as soon as a consent is revoked, data immediately stop being collected. What will happen to the data that were already collected depends on the study protocol designed by the researcher. If data deletion is needed, the researcher will have to delete the data manually from their own database (which is not related to blockchain in any way; as mentioned, blockchain only stores the information on consent and not the data as such). In this way, a blockchain-consent platform can facilitate the process of consent management and mitigate some trust issues, but it is still up to researchers to ensure correct and ethical protocols and guidelines are being followed.

Hu-manity.co developed a mobile app called My31, built on IBM blockchain, to help individuals manage their consent to the use of their personal and health information. Users can consent to sharing their data with third parties and researchers, and receive compensation for it [89,90]. Another blockchain-powered platform for managing consent is being developed by Bitfury, with the goal of managing consent for research. All updates related to user consent are timestamped and recorded on the blockchain for future auditing [91,92]. The solution can be used both as a new system and in conjunction with existing systems. HealthVerity is also developing a blockchain solution to manage consent. Unlike other solutions, HealthVerity's consent platform seems to be more focused on consumer applications that collect health data than medical or clinical research [93].

Although not specifically on consent management, DeepMind (Google's artificial intelligence conglomerate) is developing a project to allow hospitals and patients to track events related to health data in real time [94-98]. Any interaction with a patient's health data is recorded on a distributed ledger, which will store information stating that the data were used and their purpose. This project, Verifiable Audit Trail, has the potential to increase 
transparency in health research and minimize some of the trust issues between stakeholders [94-98].

There have also been several academic initiatives exploring the use of blockchain for consent management. For example, researchers from Institut National de la Santé Et de la Recherche Médicale and Assistance Publique-Hôpitaux de Paris in France have created a proof of concept to manage patient consent in clinical trials that use cryptographic signatures for e-signing. The timestamped consent for different form versions is recorded on the blockchain as a master document [14]. In Canada, Queen's University has also developed a similar solution for clinical trials, titled BlockTrial, where patients assign permission for data access and researchers can query off-chain data [99]. The Toronto-based University Health Network, in partnership with IBM and digital health agencies, is working on the patient control and consent blockchain initiative to allow the permissioned access of data, managed by patients through individual consent and built on blockchain to allow immutable storage of consent directives. Patients can access a mobile app, managing who has access to their data and for what reason. Currently, only University Health Network-produced data are available, but the goal is to enable the integration of data from multiple sources [100-102].

Velmovitsky et al [129] also developed a proof-of-concept blockchain to help patients manage consent, focusing on third-party consumer apps that collect health data such as smart devices. In this prototype, patients can grant and revoke consent for different data types and different periods (eg, users give consent for temperature to be collected but not movement, from September to October). This work uses Hyperledger Fabric to create a blockchain network and proposes a governance structure to collect data from smart devices.

\section{Discussion}

\section{Blockchain in Health Care}

The health care challenges in the areas of EHRs, supply chains, health insurance, genomics, and consent management are not mutually exclusive. For example, the use of blockchain to address challenges in genomics and EHRs shares many similar challenges regarding data ownership, data sharing, and the creation of a marketplace where owners and buyers can trade data. Consent management and EHRs can also be complementary, with blockchain-enabled solutions for consent acting as a sort of access control. Researchers and health care providers could potentially access individual health records stored in off-chain databases through blockchain infrastructure, provided that they have consent from the individual.

It is interesting to note that many of the solutions described seem to consider only an ideal workflow. In an emergency situation where the patient is unable to authorize data sharing, there should be mechanisms for doctors and nurses to access data [18].

Similar to any new technology, while blockchain seems to hold the potential to improve several existing challenges in health care and give more agency to patients, the actual impact of the technology is unclear. Many solutions want to eliminate intermediaries (eg, allowing patients and providers to connect without the need of insurance companies; however, it is impossible to determine whether this kind of disruption will actually happen and whether patients are ready for such complexity $[6,12,18])$. Although blockchain solutions can minimize inefficiencies, they are more likely to run in conjunction with existing systems from third parties. In other words, the solutions will incorporate third parties and make the process more transparent for the participants, thereby increasing trust [12]. This would also ease the adoption of blockchain in the industry, as several stakeholders would offer less resistance.

Most of the solutions we found, with a few exceptions, seem to be at the initial development or prototyping stage: their architecture and basic functionalities are planned out, and companies are now looking to raise funds and continue their implementation. Although this shows that the health care sector is positive about the potential of blockchain, it also makes it difficult to concretely evaluate the potential of the technology.

\section{Immutability, Decentralization, and Trust}

Blockchain is designed to be distributed, transparent, and immutable by design $[6,12]$ and can improve trust among stakeholders $[6,12,18,108]$. This is true in the scenarios described above. By eliminating centralized decision making, automating processes, and increasing transparency in data collection and use, blockchain can increase trust in health care processes.

However, blockchain's embedded features could prove to be a challenge if misused. For example, the fact that every node in the network maintains a copy of the ledger is redundant. Decentralized systems are less efficient, scalable, and cost-effective compared with centralized systems [12]. By design, every participant node must possess a full copy of the distributed ledger; as the number of participants increases, so does the computational requirements such as storage and energy. Blockchain has been seen as a potential solution to address issues in the environment, such as creating a marketplace for energy trading. However, some critics point out that blockchain will do more harm than good because it expends a huge amount of energy $[6,99]$. These factors may limit the scalability of the blockchain solutions. They are not limitations of the technology per se, but design characteristics, and developers must consider a trade-off between these and the desired transparency and security provided by the distributed ledger. Trade-offs between different blockchain features must also be considered (eg, using a public or permissioned blockchain), as explored by O’Donoghue et al [20].

Similarly, blockchain's immutability means it is append-only for public implementation [12]. Companies dealing with General Data Protection Regulation must abide by a key principle of regulation, which is the right to data deletion $[12,19,131]$. However, this is not an option for public blockchain implementations [12]. This is one of the reasons why it is not recommended to store personal data on the blockchain $[7,19]$. Rather, it should be stored in off-chain databases with reference to the chain. A study conducted by Park et al [132] studied the feasibility of storing, sharing, and managing records on the blockchain. The study confirmed that it was possible to manage 
records in a private blockchain, but several challenges need to be addressed first, such as data size and costs.

Although blockchain provides an immutable ledger, it should also be noted that the information stored in the ledger is only as accurate as its input. This means that developers should create mechanisms to ensure that the correct data are uploaded to the blockchain $[18,99]$.

\section{Key and Identity Management}

Information stored on the blockchain is secured through cryptographic techniques that require the use of public and private keys [18]. Private keys act as a password that allows the user to access information. For example, in the Bitcoin blockchain, user A can send Bitcoins to user B using B's public key. However, user B will only be able to access the Bitcoin with their private key. Users have one key to access all their blocks (analogous to a password to access all user data). If the key is compromised, all user data may be leaked. In the case of health care, which deals with sensitive data, this issue should not be understated [18]. For blockchain-EHR solutions, if a malicious party obtains a patient's private key, there is a significant risk of identity theft, as they will have access to the patient's full medical history [18].

One possible alternative to key management is through identity verification using blockchain [18,62,131]; it can provide an immutable ledger that stores and maintains legal documents such as birth certificates and business contracts. A person would then be able to prove their identity by accessing the blockchain [18]. Many countries, such as Estonia, use blockchain to verify various attributes of its citizens $[12,131]$. By being able to say if a person is an Estonian resident through blockchain, the platform can provide additional web-based services (eg, allowing citizens to vote on the web). The country already has an EHR system, accessed by a unique ID that uses blockchain to ensure integrity $[10,131]$. PokitDok is studying the application of a protocol called the contextually relevant identity management protocol with DokChain, in which different aspects of an identity are used for validation. For example, to buy an alcoholic a drink, the only necessary information is age. DokChain's solution would allow users to decide which specific personal information to share based on the context. To validate an identity, the technology is planning to create a consensus mechanism in which an individual's private key is partitioned among third parties. The key can be regenerated by using a subset of partitions. This is initially used for key recovery, but the goal is to have this function as implicit identity verification every time an identity needs to be validated [62]. A similar solution that distributes a user's identity attributes is being developed by the Canadian company SecureKey [131].

\section{Conclusions}

Blockchain, an immutable ledger in which all participants view an immutable version of the truth, is a promising technology that can help to minimize several challenges currently experienced in health care. This paper focused on a literature review and market assessment to determine the main challenges that could be improved by blockchain in today's health care industry. The results showed the following five challenges:
- Health records are stored between different providers in systems that lack interoperability. This makes it difficult for stakeholders to share data and, therefore, to have a complete view of a patient's health.

- Provenance and counterfeit products in drug supply chains are among the biggest hurdles faced by pharmaceutical companies today.

- There is a lack of connectivity among payers, providers, and patients in terms of insurance, which prevents access and coordination of care.

- Genomics data, one of the greatest promises of precision health, are limited by high costs, lack of massive data sets, and interoperability issues.

- Increasingly advanced methods of data collection and analysis raise concerns regarding ethics, ownership, privacy, and regulations of health data.

Although solutions vary, blockchain can provide an immutable and tamperproof log of transactions. Blockchain's features of immutability, decentralization, distribution, and transparency can optimize processes, minimize inefficiencies, and increase trust in all contexts covered in this review. However, no silver bullet to solve every need in health care exists, and companies, developers, and decision makers need to be careful when considering a blockchain solution. Although the technology has potential, it also brings new concerns about data ownership and security.

\section{Limitations}

As the aim of this review was to provide an industry point of view of the use of blockchain in health care, when defining the protocols for inclusion and exclusion, we did not include a critical component to evaluate the solutions included. Furthermore, while we searched for white papers and reports of the solutions whenever possible, we included in our results solutions already in place, in early stages of development, or recently announced, as our goal was to provide a mindset of the health care industry pertaining to blockchain. Future work should focus on critically analyzing the solutions described here for feasibility and efficiency. An interesting consideration from this analysis would be to define which challenges can be further improved with the use of blockchain. In other words, this analysis could point to where blockchain would be the most useful. In addition, blockchain can be used in conjunction with new fields of computer science, including big data and artificial intelligence [6,117]. Future work should also focus on studying blockchain in the context of these emerging technologies. Finally, it is important to note that the ontology used here, dividing the topics into five major challenges, is not exhaustive. There are many use cases where blockchain can be applied that, while not directly related to health care, may affect it (eg, key and identity management). In addition, many challenges described in the literature (eg, interoperability and data sharing of health systems) are encompassed by this ontology. In exploring these five areas, our aim was to provide an overview of the solutions being developed by the health care industry and which areas they are directing their efforts, rather than providing a defining list of use cases in health care in which blockchain can be used. 


\section{Acknowledgments}

This research was supported by the Canadian Standards Association Group and MITACS internship to the corresponding author.

\section{Authors' Contributions}

PEV wrote the manuscript and conducted a literature review. PEV, PPM, LXF, and FMB contributed to the conceptualization, design, and approach of the manuscript, as well as to the interpretation of the argument made in the manuscript. All authors contributed to the writing and revision of the manuscript. All authors provided the final approval of the manuscript and agree to be accountable for this manuscript.

\section{Conflicts of Interest}

None declared.

\section{References}

1. Prosperi M, Min JS, Bian J, Modave F. Big data hurdles in precision medicine and precision public health. BMC Med Inform Decis Mak 2018 Dec 29;18(1):139. [doi: 10.1186/s12911-018-0719-2] [Medline: 30594159]

2. de Arriba-Pérez F, Caeiro-Rodríguez M, Santos-Gago J. Collection and processing of data from wrist wearable devices in heterogeneous and multiple-user scenarios. Sensors (Basel) 2016 Sep 21;16(9):1538 [FREE Full text] [doi:

10.3390/s16091538] [Medline: 27657081]

3. Mooney SJ, Pejaver V. Big data in public health: terminology, machine learning, and privacy. Annu Rev Public Health 2018 Apr 01;39(1):95-112 [FREE Full text] [doi: 10.1146/annurev-publhealth-040617-014208] [Medline: 29261408]

4. Sakr S, Elgammal A. Towards a comprehensive data analytics framework for smart healthcare services. Big Data Res 2016 Jun;4:44-58. [doi: 10.1016/j.bdr.2016.05.002]

5. Barrett MA, Humblet O, Hiatt RA, Adler NE. Big data and disease prevention: from quantified self to quantified communities. Big Data 2013 Sep;1(3):168-175. [doi: 10.1089/big.2013.0027] [Medline: 27442198]

6. Bublitz FM, Oetomo A, Sahu KS, Kuang A, Fadrique LX, Velmovitsky PE, et al. Disruptive technologies for environment and health research: an overview of artificial intelligence, blockchain, and internet of things. Int J Environ Res Public Health 2019 Oct 11;16(20):3847 [FREE Full text] [doi: 10.3390/ijerph16203847] [Medline: 31614632]

7. Azaria A, Ekblaw A, Vieira T, Lippman A. MedRec: Using blockchain for medical data access and permission management. In: Proceedings of the 2nd International Conference on Open and Big Data (OBD). 2016 Presented at: 2nd International Conference on Open and Big Data (OBD); Aug. 22-24, 2016; Vienna, Austria p. 30. [doi: 10.1109/obd.2016.11]

8. Ekblaw AC. MedRec: blockchain for medical data access, permission management and trend analysis. Massachusetts Institute of Technology. 2017. URL: https://dspace.mit.edu/bitstream/handle/1721.1/109658/ 987247095-MIT.pdf?sequence=1\%0Ahttps://dspace.mit.edu/handle/1721.1/109658 [accessed 2021-05-22]

9. Grishin D, Obbad K, Estep P, Cifric M, Zhao Y, Church G. Blockchain-enabled genomic data sharing and analysis platform. 2018. URL: https://arep.med.harvard.edu/pdf/Grishin Church v4.52 2018.pdf [accessed 2021-05-22]

10. Healthcare rallies for blockchains. IBM Institute for Business Value. 2017. URL: https://www.ibm.com/downloads/cas/ BBRQK3WY [accessed 2021-05-22]

11. Team Medicine: how life sciences can win with blockchain. IBM Institute for Business Value. URL: $\underline{\text { https://www.ibm.com/ }}$ downloads/cas/RYD0QA7G [accessed 2021-05-22]

12. Urban MC, Pineda D. Inside the black blocks. Toronto, ON: Mowat Centre; 2018. URL: https://munkschool.utoronto.ca/ mowatcentre/wp-content/uploads/publications/168 inside the black blocks.pdf [accessed 2021-05-25]

13. Farouk A, Alahmadi A, Ghose S, Mashatan A. Blockchain platform for industrial healthcare: vision and future opportunities. Comp Commun 2020 Mar;154:223-235. [doi: 10.1016/j.comcom.2020.02.058]

14. Benchoufi M, Ravaud P. Blockchain technology for improving clinical research quality. Trials 2017 Jul 19;18(1):335 [FREE Full text] [doi: 10.1186/s13063-017-2035-z] [Medline: 28724395]

15. Hollander JE, Carr BG. Virtually perfect? Telemedicine for Covid-19. N Engl J Med 2020 Apr 30;382(18):1679-1681. [doi: 10.1056/nejmp2003539]

16. Dragov R, Croce CL, Hefny M. How blockchain can help in the COVID-19 crisis and recovery. IDC UK Blog. 2020. URL: https://blog-idcuk.com/blockchain-help-in-the-covid-19-and-recovery/ [accessed 2020-05-20]

17. World Economic Forum: how blockchain could help with Covid-19 supply chain disruption. Ledger Insights. 2020. URL: https://www.ledgerinsights.com/world-economic-forum-how-blockchain-could-help-with-covid-19-supply-chain-disruption/ [accessed 2020-05-25]

18. McGhin T, Choo KR, Liu CZ, He D. Blockchain in healthcare applications: research challenges and opportunities. J Net Comp Appl 2019 Jun;135:62-75. [doi: 10.1016/j.jnca.2019.02.027]

19. Vazirani AA, O'Donoghue O, Brindley D, Meinert E. Implementing blockchains for efficient health care: systematic review. J Med Internet Res 2019 Feb 12;21(2):e12439 [FREE Full text] [doi: 10.2196/12439] [Medline: 30747714] 
20. O'Donoghue O, Vazirani AA, Brindley D, Meinert E. Design choices and trade-offs in health care blockchain implementations: systematic review. J Med Internet Res 2019 May 10;21(5):e12426 [FREE Full text] [doi: 10.2196/12426] [Medline: $\underline{31094344]}$

21. Hasselgren A, Kralevska K, Gligoroski D, Pedersen SA, Faxvaag A. Blockchain in healthcare and health sciences-a scoping review. Int J Med Inform 2020 Feb;134:104040 [FREE Full text] [doi: 10.1016/j.ijmedinf.2019.104040] [Medline: 31865055]

22. Chukwu E, Garg L. A systematic review of blockchain in healthcare: frameworks, prototypes, and implementations. IEEE Access 2020;8:21196-21214. [doi: 10.1109/access.2020.2969881]

23. Agbo C, Mahmoud Q, Eklund J. Blockchain technology in healthcare: a systematic review. Healthcare (Basel) 2019 Apr 04;7(2):56 [FREE Full text] [doi: 10.3390/healthcare7020056] [Medline: $\underline{30987333}$ ]

24. Reviews: from systematic to narrative: narrative review. University of Alabama - Birmingham. URL: https://guides. library.uab.edu/c.php?g=63689\&p=409774 [accessed 2020-05-25]

25. Levac D, Colquhoun H, O'Brien KK. Scoping studies: advancing the methodology. Implement Sci 2010 Sep 20;5(1):69 [FREE Full text] [doi: 10.1186/1748-5908-5-69] [Medline: 20854677]

26. Leader in blockchain news. CoinDesk. URL: https://www.coindesk.com/ [accessed 2020-05-18]

27. Cointelegraph. URL: https://cointelegraph.com/ [accessed 2020-05-18]

28. Get smarter about what matters to you. Medium. URL: https://medium.com/ [accessed 2020-05-18]

29. MedRec. URL: https://medrec.media.mit.edu/ [accessed 2018-10-03]

30. PatientTruth. Embleema - Blockchain for Real-World Evidence. URL: https://embleema.com/2020/01/16/ explore-real-world-evidence/ [accessed 2018-11-08]

31. Embleema blockchain network v.2. Embleema WhitePaper. 2018. URL: https://icocube.io/uploads/Embleema.pdf [accessed 2021-05-22]

32. Adams J. CareX Whitepaper. 2018. URL: https://coins.newbium.com/post/13327-carex-blockchain-healthcare-ecosystem [accessed 2021-05-22]

33. Stoffregen E. Blockchain healthcare ecosystem in 2018. Medium. 2018. URL: https://medium.com/@erikstoffregen/ blockchain-healthcare-ecosystem-d21631024454 [accessed 2018-11-22]

34. Kovach A, Ronai G. MyMEDIS: a new medical data storage and access system. 2018. URL: https://mymedis.in/documents/ MEDIS-White-Paper.pdf [accessed 2021-05-22]

35. Globally decentralized medical data store and blockchain-based ecosystem. MEDIS. URL: https://mymedis.in/ [accessed 2019-03-12]

36. Allison I. Gem shows off first blockchain application for health claims. Intenational Business Times. 2017. URL: https:/ /www.ibtimes.co.uk/gem-shows-off-first-blockchain-application-health-claims-1622574 [accessed 2018-10-09]

37. Health. Gem. URL: https://enterprise.gem.co/health/ [accessed 2018-10-04]

38. Rizzo P. Gem partners with Philips for blockchain healthcare initiative. CoinDesk. 2016. URL: $\underline{\text { https://www.coindesk.com/ }}$ gem-philips-blockchain-healthcare/ [accessed 2018-10-05]

39. Redman J. Gem health unveils medical management blockchain platform. Bitcoin. 2016. URL: https://news.bitcoin.com/ gem-health-blockchain-medical-mgmt/ [accessed 2018-10-09]

40. Shieber J. Gem looks to CDC and European giant Tieto to take blockchain into healthcare. TechCrunch. 2017. URL: https:/ /techcrunch.com/2017/09/25/gem-looks-to-cdc-and-european-giant-tieto-to-take-blockchain-into-healthcare/ [accessed 2018-10-09]

41. Albeyatti A. Whitepaper. MedicalChain. 2017. URL: https://medicalchain.com/en/whitepaper/ [accessed 2021-05-22]

42. Blockchain for electronic health records. MedicalChain. URL: https://medicalchain.com/en/ [accessed 2019-02-27]

43. Rebuilding healthcare for the next generation. Citizen Health. URL: https://citizenhealth.io/ [accessed 2018-11-22]

44. Humantiv. A Citizen Health Development. URL: https://citizenhealth.io/humantiv/ [accessed 2018-11-22]

45. Medoplex. Citizen Health. URL: https://citizenhealth.io/medoplex/ [accessed 2018-11-22]

46. Siwicki B. The next big thing in pharmacy supply chain: Blockchain. Healthcare IT News. 2017. URL: https://www. healthcareitnews.com/news/next-big-thing-pharmacy-supply-chain-blockchain [accessed 2018-10-09]

47. Block Verify. URL: https://true.global/startups/blockverify/ \#: :text=Blockverify\%20is\%20a\%20blockchain\%2Dbased,\%2C\%20apparel\%2C\%20electronics\%20and\%20pharmaceuticals [accessed 2018-10-09]

48. Hulseapple C. Block verify uses blockchains to end counterfeiting and 'make world more honest'. Cointelegraph. 2015. URL: https://cointelegraph.com/news/block-verify-uses-blockchains-to-end-counterfeiting-and-make-world-more-honest [accessed 2018-10-09]

49. De N. Pharma giant merck eyes blockchain for fighting counterfeit meds. CoinDesk. 2018. URL: https://www.coindesk.com/ merck-proposes-blockchain-platform-for-combat-counterfeiters/ [accessed 2018-11-07]

50. Haring B. BlockTribune. URL: https://blocktribune.com/blockchain-patent-filed-by-pharmaceutical-giant-merck-co/ [accessed 2018-11-07]

51. Modum. URL: https://modum.io/ [accessed 2018-10-09]

52. Products. Modum. URL: https://www.modum.io/solutions [accessed 2018-10-09] 
53. Data integrity for supply chain operations, powered by blockchain technology. Modum. 2017. URL: https://assets.modum.io/ wp-content/uploads/2017/08/modum-whitepaper-v.-1.0.pdf [accessed 2021-05-22]

54. Uhlmann S. Reducing counterfeit products with blockchains. University of Zurich. 2017. URL: https://www.merlin.uzh.ch/ contributionDocument/download/10024 [accessed 2021-05-22]

55. Stanley A. Ready to rumble: IBM launches food trust blockchain for commercial use. Forbes. 2018. URL: https://www. forbes.com/sites/astanley/2018/10/08/ready-to-rumble-ibm-launches-food-trust-blockchain-for-commercial-use/ \#68bf18817439 [accessed 2018-10-18]

56. IBM food trust expands blockchain network to foster a safer, more transparent and efficient global food system. IBM News Room. URL: https://newsroom.ibm.com/

2018-10-08-IBM-Food-Trust-Expands-Blockchain-Network-to-Foster-a-Safer-More-Transparent-and-Efficient-Global-Food-System-1 [accessed 2018-10-18]

57. IBM Food Trust: a new era for the world's food supply. 2018. URL: https://www.ibm.com/blockchain/solutions/food-trust [accessed 2021-05-22]

58. Walmart's food safety solution using IBM food trust built on the IBM blockchain platform. IBM Blockchain. 2017. URL: https://www.youtube.com/watch?time continue=173\&v=SV0KXBXSoio [accessed 2018-09-26]

59. Huillet M. Alibaba's Ant Financial to launch blockchain backend-as-a-service platform. Cointelegraph. 2018. URL: https:/ /cointelegraph.com/news/alibabas-ant-financial-to-launch-blockchain-backend-as-a-service-platform [accessed 2018-10-19]

60. Zhao W. Ant Financial is launching a blockchain app to tackle food fraud. CoinDesk. 2018. URL: https://www.coindesk.com/ ant-financial-is-launching-a-blockchain-app-to-tackle-food-fraud/ [accessed 2018-09-26]

61. DokChain. PokitDok. URL: https://pokitdok.com/dokchain/ [accessed 2018-09-26]

62. Smith WB. DokChain: intelligent automation in healthcare transaction processing. PokitDok. 2018. URL: https://pokitdok. com/wp-content/uploads/2018/02/DokChain_Whitepaper.pdf [accessed 2021-05-22]

63. Brennan B. DokChain by PokitDok - blockchain for healthcare. Blockchain Healthcare Review. URL: $\underline{\text { https:/ }}$ /blockchainhealthcarereview.com/dokchain-by-pokitdoc-blockchain-for-healthcare/ [accessed 2018-10-01]

64. Healthcare claims processing software. PokitDok. URL: https://pokitdok.com/business/claims-management/ [accessed 2018-10-03]

65. Autonomous Auto-Adjudication 101: blockchains in healthcare. Pokitdok. URL: https://techcrunch.com/2017/05/10/ pokitdok-teams-with-intel-on-healthcare-blockchain-solution/ [accessed 2018-10-03]

66. Healthcare propensity to pay. PokitDok. URL: https://pokitdok.com/business/payment-risk/ [accessed 2018-10-02]

67. Patient access solutions. PokitDok. URL: https://pokitdok.com/business/patient-access-solutions/ [accessed 2018-10-03]

68. Healthcare reimbursement solutions - hospital payment systems. Payspan. URL: https://payspan.com/ [accessed 2018-11-08]

69. How blockchain can connect payers, providers and consumers. Payspan. URL: https://payspan.com/wp-content/uploads/ 2018/02/Payspan-white-paper-February-2018.pdf [accessed 2021-05-22]

70. Nebula Genomics. URL: https://www.nebula.org/ [accessed 2018-11-12]

71. LunaDNA : frequently asked questions. URL: https://support.lunadna.com/support/solutions/articles/ 43000038763-what-is-lunadna- [accessed 2018-11-12]

72. About LunaDNA - learn more about the LunaDNA team company. URL: https://www.lunadna.com/what-we-do/ [accessed 2018-11-12]

73. Interview with Luna DNA's Co-Founder and President Dawn Barry. SanDiegOmics. 2018. URL: https://sandiegomics.com/ luna-dna-interview-with-co-founder-dawn-barry/ [accessed 2018-11-12]

74. Bigelow B. Luna DNA uses blockchain to share genomic data as a "Public Benefit". Xconomy. 2018. URL: https://xconomy. com/san-diego/2018/01/22/luna-dna-uses-blockchain-to-share-genomic-data-as-a-public-benefit/ [accessed 2018-11-12]

75. Farr C, Levy A. Luna Coin project: sell your genetic data for crypto tokens. CNBC Tech. 2017. URL: https://www.cnbc.com/ 2017/12/18/luna-coin-project-sell-your-genetic-data-for-crypto-tokens.html [accessed 2018-11-12]

76. Project SHIVOM (Official Video) - powering the next era of genomics through blockchain. Shivom. 2018. URL: https:/ /www.youtube.com/watch?v=jce9vB5zbps [accessed 2018-11-14]

77. Shivom. URL: https://shivom.io/ [accessed 2018-11-14]

78. Thrill W. Shivom: the uncanny synergy of blockchain and genomics. Hacker Noon. 2018. URL: https://hackernoon.com/ shivom-the-uncanny-synergy-of-blockchain-and-genomics-e1ca7f2a0173 [accessed 2018-11-15]

79. Shivom innovation council. Shivom. URL: https://www.youtube.com/watch?v=NuaOV82kCjc [accessed 2018-11-14]

80. Zenome. URL: https://zenome.io/ [accessed 2018-11-15]

81. Kulemin N, Popov S, Gorbachev A. The Zenome Project: whitepaper blockchain-based genomic ecosystem. Zenome.io 2017:A. [doi: 10.13140/RG.2.2.25865.13925]

82. Thrill W. EncrypGen uses blockchain technology to store and manage DNA profiles. Hacker Noon. URL: https://hackernoon. com/encrypgen-uses-blockchain-technology-to-store-and-manage-dna-profiles-a920e898b6a8 [accessed 2018-11-15]

83. Gene-chain DNA data marketplace. EncrypGen. URL: https://encrypgen.com/encrypgen-gene-chain-dna-data-marketplace/ [accessed 2018-11-15]

84. Marketplace partners. EncrypGen. URL: https://encrypgen.com/marketplace-partners/ [accessed 2018-11-15]

85. The DNA data marketplace. EncrypGen. URL: https://encrypgen.com/ [accessed 2018-11-15] 
86. S Korea's Macrogen to leverage blockchain for genomic data. Cryptovest. 2018. URL: https://www.investing.com/news/ cryptocurrency-news/s-koreas-macrogen-to-leverage-blockchain-for-genomic-data-1562879 [accessed 2018-10-10]

87. Say N. Macrogen develops blockchain platform to share genetic data. Blockonomi. 2018. URL: https://blockonomi.com/ macrogen-blockchain/ [accessed 2018-10-10]

88. Ji-young S. Korea's Macrogen, Bigster to create blockchain-based medical data platform. The Korea Herald. 2018. URL: http://www.koreaherald.com/view.php?ud=20180806000646 [accessed 2018-10-10]

89. Hu-manity.co collaborates with IBM blockchain on consumer app to manage personal data property rights. IBM Announcements. 2018. URL: https://newsroom.ibm.com/

2018-09-06-Hu-manity-co-Collaborates-with-IBM-Blockchain-on-Consumer-App-to-Manage-Personal-Data-Property-Rights [accessed 2020-05-15]

90. Takahashi D. Hu-manity.co uses IBM blockchain to give you the right to control your personal data. VentureBeat. URL: https://venturebeat.com/2018/09/06/hu-manity-co-uses-ibm-blockchain-to-give-you-the-right-to-control-your-personal-data/ [accessed 2020-05-15]

91. Alexandre A. New Bitfury joint project to manage medical data permissions with blockchain tech. Cointelegraph. 2019. URL: https://cointelegraph.com/news/new-bitfury-joint-project-to-manage-medical-data-permissions-with-blockchain-tech [accessed 2020-05-15]

92. Bitfury announces blockchain-based consent management system; partners with Hancom to distribute Crystal platform. TokenPost. 2019. URL: https://tokenpost.com/

Bitfury-announces-blockchain-based-consent-management-system-partners-with-Hancom-to-distribute-Crystal-platform-1603 [accessed 2020-05-15]

93. HealthVerity consent. URL: https://healthverity.com/solutions/healthverity-consent/ [accessed 2020-05-21]

94. Hern A. Google's DeepMind plans bitcoin-style health record tracking for hospitals. The Guardian. 2017. URL: https:/ /www.theguardian.com/technology/2017/mar/09/google-deepmind-health-records-tracking-blockchain-nhs-hospitals [accessed 2018-09-28]

95. Suleyman M, Laurie B. Trust, confidence and verifiable data audit. DeepMind. 2017. URL: https://deepmind.com/blog/ trust-confidence-verifiable-data-audit/ [accessed 2021-05-22]

96. DeepMind. URL: https://deepmind.com/ [accessed 2021-05-22]

97. Metz C. Google DeepMind's untrendy play to make the blockchain actually useful. Wired. 2017. URL: https://www. wired.com/2017/03/google-deepminds-untrendy-blockchain-play-make-actually-useful/ [accessed 2018-09-28]

98. Powles J, Hodson H. Google DeepMind and healthcare in an age of algorithms. Health Technol (Berl) $2017 \mathrm{Mar}$ 16;7(4):351-367 [FREE Full text] [doi: 10.1007/s12553-017-0179-1] [Medline: 29308344]

99. Maslove DM, Klein J, Brohman K, Martin P. Using blockchain technology to manage clinical trials data: a proof-of-concept study. JMIR Med Inform 2018 Dec 21;6(4):e11949 [FREE Full text] [doi: 10.2196/11949] [Medline: 30578196]

100. Wiljer D, Brudnicki S. Bringing blockchain to healthcare for a new view on data. IBM Think Blog. 2019. URL: https:/ /www.ibm.com/blogs/think/2019/08/bringing-blockchain-to-healthcare-for-a-new-view-on-data/ [accessed 2020-05-25]

101. Canadian hospital collaborates with IBM for health consent blockchain. Ledger Insights. 2020. URL: https://www. ledgerinsights.com/health-consent-blockchain-university-health-network-uhn/ [accessed 2020-05-25]

102. Smith K. Clinician engagement, local impact awards, budget risk meetings and beyond. University Health Network. 2019. URL: https://www.uhn.ca/corporate/AboutUHN/Updates from CEO/Pages/

Clinician_engagement_Local_Impact_Awards_budget_risk_meetings_and_beyond.aspx [accessed 2020-05-25]

103. Velmovitsky P, Morita P. Blockchain platform for consent management in ambient assisted living. AAL Forum Poster Presentations. 2019. URL: https://www.aalforum.eu/about/poster-presentations-aal-forum-2019/ [accessed 2020-05-26]

104. Guegan D. Public blockchain versus private blockhain. HAL Archives-Ouvertes. 2017. URL: https://halshs. archives-ouvertes.fr/halshs-01524440/document [accessed 2021-05-22]

105. Dias JP, Ferreira HS, Martins A. A blockchain-based scheme for access control in e-health scenarios. In: Proceedings of the Tenth International Conference on Soft Computing and Pattern Recognition (SoCPaR 2018). Switzerland: Springer; 2018:238-247.

106. Abouelmehdi K, Beni-Hssane A, Khaloufi H, Saadi M. Big data security and privacy in healthcare: a review. Procedia Comp Sci 2017;113:73-80. [doi: 10.1016/j.procs.2017.08.292]

107. Blockchain's healthcare technology impact. Cognizant. URL: https://soundcloud.com/cognizant-worldwide/ impact-of-blockchain-technology-on-healthcare-codex2937 [accessed 2018-11-09]

108. Gorenflo C, Golab L, Keshav S. Mitigating trust issues in electric vehicle charging using a blockchain. In: Proceedings of the Tenth ACM International Conference on Future Energy Systems. 2019 Presented at: e-Energy '19: The Tenth ACM International Conference on Future Energy Systems; June, 2019; Phoenix AZ USA p. 160-164. [doi: $10.1145 / 3307772.3328283]$

109. Electronic health records. Canada Health Infoway. URL: https://www.infoway-inforoute.ca/en/solutions/ digital-health-foundation/electronic-health-records [accessed 2018-09-27]

110. Sharma R. Blockchain: the magic pill to alleviate the pain points of the healthcare industry? France Canada Chamber of Commerce Ontario. 2018. URL: https://www.fccco.org/post/blockchain-in-healthcare [accessed 2021-05-22] 
111. Velmovitsky PE, Miranda PA, Fadrique LX, Morita PP. Blockchain in health care. CSA Group Report. 2021. URL: https:/ /www.csagroup.org/article/research/blockchain-in-health-care/ [accessed 2021-04-06]

112. Stanley A. Better off abroad? Blockchain health firms gain ground outside the US. CoinDesk. URL: https://www. coindesk.com/better-off-abroad-blockchain-health-firms-are-gaining-ground-outside-the-us/ [accessed 2018-10-09]

113. Tieto establishes a blockchain pilot program in the Nordics - introduces a global identity network for secure digital interactions. Tietoevry Newsroom. URL: https://www.tietoevry.com/en/newsroom/all-news-and-releases/press-releases/ 2017/11/ tieto-establishes-a-blockchain-pilot-program-in-the-nordics--introduces-a-global-identity-network-for-secure-digital-in/ [accessed 2018-10-09]

114. ICO alert report: BlockRx. URL: https://blog.icoalert.com/ico-alert-report-blockrx [accessed 2018-11-07]

115. Drug Supply Chain Security Act (DSCSA). US Food \& Drug Administration. URL: https://www.fda.gov/drugs/ drug-supply-chain-integrity/drug-supply-chain-security-act-dscsa [accessed 2020-02-01]

116. The Drug Supply Chain Security Act and Blockchain : a white paper for stakeholders in the pharmaceutical supply chain. Center for Supply Chain Studies. 2018. URL: https://static1.squarespace.com/static/563240cae4b056714fc21c26/t/ 5b3426b088251b230ba9e6e5/1530144436146/C4SCS+White+Paper +DSCSA+and+Blockchain+Study FINAL3.pdf [accessed 2021-05-22]

117. Building block(chain)s for a better planet. PwC Global. 2018. URL: https://www.pwc.com/gx/en/services/sustainability/ building-blockchains-for-the-earth.html [accessed 2021-05-22]

118. What is a gene? MedlinePlus. URL: https://ghr.nlm.nih.gov/primer/basics/gene [accessed 2020-05-12]

119. Ferguson GK. Primer. The Human Genome: Poems on the Book of Life. URL: http://www.thehumangenome.co.uk/ THE HUMAN GENOME/Primer.html [accessed 2020-05-12]

120. Barry D. There is nothing more personal than your genome. Tedx Talks. 2016. URL: https://www.youtube.com/ watch?v=M3SLHhWYxiY [accessed 2018-11-12]

121. Nebula genomics - platform overview. Vimeo. URL: https://vimeo.com/287152656 [accessed 2018-11-12]

122. Adams SA, Petersen C. Precision medicine: opportunities, possibilities, and challenges for patients and providers. J Am Med Inform Assoc 2016 Jul;23(4):787-790. [doi: 10.1093/jamia/ocv215] [Medline: 26977101]

123. Khoury MJ. The shift from personalized medicine to precision medicine and precision public health: words matter!. Centers for Disease Control and Prevention. 2016. URL: https://blogs.cdc.gov/genomics/2016/04/21/shift/ [accessed 2019-08-08]

124. Benke K, Benke G. Artificial Intelligence and Big Data in Public Health. Int J Environ Res Public Health 2018 Dec 10;15(12):2796 [FREE Full text] [doi: 10.3390/ijerph15122796] [Medline: 30544648]

125. Barney JR, Antisdel M. Common problems in informed consent. Yale University. 2013. URL: https://your.yale.edu/ policies-procedures/other/common-problems-informed-consent [accessed 2021-05-19]

126. Novitzky P, Smeaton AF, Chen C, Irving K, Jacquemard T, O'Brolcháin F, et al. A review of contemporary work on the ethics of ambient assisted living technologies for people with dementia. Sci Eng Ethics 2015 Jun 19;21(3):707-765. [doi: 10.1007/s11948-014-9552-x] [Medline: 24942810]

127. Gupta U. Informed consent in clinical research: revisiting few concepts and areas. Perspect Clin Res 2013 Jan;4(1):26-32 [FREE Full text] [doi: 10.4103/2229-3485.106373] [Medline: 23533976]

128. Morgan-Linnell SK, Stewart DJ, Kurzrock R. U.S. Food and Drug Administration Inspections of Clinical Investigators: overview of results from 1977 to 2009. Clin Cancer Res 2014 Apr 15;20(13):3364-3370. [doi: 10.1158/1078-0432.ccr-13-3206]

129. Velmovitsky PE, Miranda PA, Vaillancourt H, Donovska T, Teague J, Morita PP. A blockchain-based consent platform for active assisted living: modeling study and conceptual framework. J Med Internet Res 2020 Dec 04;22(12):e20832 [FREE Full text] [doi: 10.2196/20832] [Medline: 33275111]

130. Art. 17 GDPR : right to erasure ('right to be forgotten'). General Data Protection Regulation (GDPR). URL: https://gdpr-info. eu/art-17-gdpr/ [accessed 2021-04-07]

131. Compert C, Luinetti M, Portier B. Blockchain and GDPR: how blockchain could address five areas associated with GDPR compliance. IBM Security. 2018. URL: https://iapp.org/media/pdf/resource center/blockchain and gdpr.pdf [accessed 2021-05-15]

132. Park YR, Lee E, Na W, Park S, Lee Y, Lee J. Is blockchain technology suitable for managing personal health records? Mixed-methods study to test feasibility. J Med Internet Res 2019 Feb 08;21(2):e12533 [FREE Full text] [doi: 10.2196/12533] [Medline: 30735142]

\section{Abbreviations}

DSCSA: Drug Supply Chain Security Act

EHR: electronic health record

RQ: research question 
Edited by C Lovis; submitted 26.05.20; peer-reviewed by M Raghavendra, A Hasselgren; comments to author 23.06.20; revised version received 21.07.20; accepted 24.04.21; published 08.06.21

Please cite as:

Velmovitsky PE, Bublitz FM, Fadrique LX, Morita PP

Blockchain Applications in Health Care and Public Health: Increased Transparency

JMIR Med Inform 2021;9(6):e20713

URL: https://medinform.jmir.org/2021/6/e20713

doi: 10.2196/20713

PMID:

CPedro Elkind Velmovitsky, Frederico Moreira Bublitz, Laura Xavier Fadrique, Plinio Pelegrini Morita. Originally published in JMIR Medical Informatics (https://medinform.jmir.org), 08.06.2021. This is an open-access article distributed under the terms of the Creative Commons Attribution License (https://creativecommons.org/licenses/by/4.0/), which permits unrestricted use, distribution, and reproduction in any medium, provided the original work, first published in JMIR Medical Informatics, is properly cited. The complete bibliographic information, a link to the original publication on https://medinform.jmir.org/, as well as this copyright and license information must be included. 